\title{
eJRIEPS
}

Ejournal de la recherche sur l'intervention en éducation physique et sport

48 | 2021

Varia

\section{Débat, conceptualisation et éducation physique et sportive}

Debate, conceptualization and physical education and sport

Zeineb Zerai, Jean-Francis Gréhaigne et Paul Godbout

\section{(2) OpenEdition}

\section{Journals}

Édition électronique

URL : http://journals.openedition.org/ejrieps/5642

DOI : 10.4000/ejrieps.5642

ISSN : 2105-0821

Éditeur

ELLIADD

\section{Référence électronique}

Zeineb Zerai, Jean-Francis Gréhaigne et Paul Godbout, « Débat, conceptualisation et éducation physique et sportive », eJRIEPS [En ligne], 48 | 2021, mis en ligne le 12 janvier 2021, consulté le 28 janvier 2021. URL : http://journals.openedition.org/ejrieps/5642; DOI : https://doi.org/10.4000/ejrieps. 5642

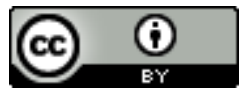

La revue eJRIEPS est mise à disposition selon les termes de la Creative Commons Attribution 4.0 International License. 


\section{Débat, conceptualisation et éducation physique et sportive}

Zeineb Zerai ${ }^{\star}$, Jean-Francis Gréhaigne** \& Paul Godbout ${ }^{\star \star \star}$

* Université de Sohar, Oman

** Université de Bourgogne Franche-Comté, France

*** Université Laval, Québec, Canada

Résumé

Cet article se propose de faire un bilan des travaux sur l'étude de cette organisation pédagogique particulière qu'est le débat d'idées. Utilisé en EPS dans l'enseignement des sports collectifs en France, il consiste en une situation dans laquelle les élèves s'expriment sur la base d'observations ou d'activités personnelles vécues dans le jeu. Le débat d'idées vise des apprentissages par le recours à la verbalisation, la pensée critique et la pratique réflexive ainsi que la construction de connaissances en situation d'opposition.

Mots clés : débat d'idées, conceptualisation, pratique réflexive, pensée tactique, sports collectifs

Debate, conceptualization and physical education and sport Summary

This paper presents a review of studies conducted with regards to a particular learning setup, the debate of ideas. Used in physical education in the context of team-sport teaching in France, the set-up consists of situations during which students express themselves. The debate of ideas promotes learning through verbalization, critical thinking, reflexive practice and the construction of knowledge in opposition situations.

Keywords: Debate-of-ideas, conceptualization, reflexive practice, tactical thinking, team sports, 


\section{Introduction}

Le débat en classe est un dispositif d'enseignement/apprentissage reconnu comme efficace, en particulier parce qu'elle favorise les interactions entre les élèves (cf. Suisse et France, Deriaz, Poussin, \& Gréhaigne, 1998 ; France, Nachon, \& Chang, 2004 ; Darnis \& Lafont, 2008 ; Belgique, Cloes, Derome, \& Bonvoisin, 2014 ; Angleterre, Harvey \& Gittins ; 2014 ; Suisse ; Lenzen \& Reylé, 2017). Ce type d'enseignement actif cultive des procédures de pensées multiformes et développe l'attention, la compréhension ainsi que l'utilisation appropriée des connaissances et compétences grâce, en particulier, à une attitude positive de la part des formés. Les études et les expériences pratiques à propos du débat d'idées se développent dans les pays francophones dans le cadre de l'enseignement de l'éducation physique et sportive (EPS) et plus particulièrement dans celui des sports collectifs. Le débat d'idées (Deriaz, Poussin, \& Gréhaigne, 1998 ; Gréhaigne \& Godbout, 1998 ; Gréhaigne \& Deriaz, 2007 ; Darnis, Lafont \& Menaut, 2007) est une organisation pédagogique qui permet une discussion, autant que possible constructive sur un problème précis, à laquelle prennent part des élèves ayant des réflexions ou des expériences différentes sur le problème considéré. Le débat a été utilisé dans différents contextes éducatifs et présente des avantages dont l'une des caractéristiques fondamentales est que les personnes qui travaillent ensemble pour atteindre le même objectif finiront par devenir plus positives mutuellement (Zerai, 2011).

En EPS, l'utilisation du débat d'idées ne se limite pas à l'enseignement / apprentissage des sports d'équipe. Les chercheurs et les praticiens ont également utilisé cet outil pédagogique avec la danse, l'athlétisme et l'acrosport. En danse, Cadopi et Bonnery (1990) ont proposé un travail de création par groupe où la future chorégraphie est débattue dans chaque groupe de danseurs. Pour sa part, Lab (2007) a exploré comment l'utilisation du débat peut motiver les élèves et les aider à améliorer leurs tactiques, leurs stratégies et leur collaboration tout en se livrant à une course de demi-fond. Enfin, Caty (2004) a utilisé le débat avec ses élèves en acrosport. Lors d'un débat d'idées, les élèves échangent sur la présentation à réaliser, les évolutions possibles, la musique voire la sécurité. Ainsi, en classe, les élèves sont plus motivés et capables d'avoir des interactions constructives lorsqu'ils ont à effectuer une tâche collective (Zerai, 2017).

Dans les sports collectifs, c'est une situation dans laquelle les élèves s'expriment et échangent des faits et des idées, sur la base d'observations ou d'activités personnelles vécues pendant le déroulement du jeu. Dans ce type de débat, l'objectif de l'argumentation est de discuter une ou plusieurs pistes pour résoudre les problèmes rencontrés dans le jeu. L'utilisation du débat dans les activités d'apprentissage est un mode didactique qui 
considère donc la classe comme une communauté regroupant enseignant et élèves, dans laquelle toute action est nécessairement conjointe (Sensevy, 2007). L'élaboration et l'évolution des actions en projet des élèves impliquent pour le professeur d'être actif et attentif aux processus en cours, voire parfois de formuler des consignes et/ou des règles de fonctionnement afin de poser des exigences visant de réels apprentissages. Pour les élèves, observation et analyse du jeu, mise en mots, interlocutions discursives et feuilles de route sont au cœur du débat. Comme indiqué par Schunk (1986), "studies demonstrate that verbalization can improve children's learning of information, modeled actions, and strategies, as well as their self-efficacy for performing tasks. Collectively, these findings support the notion that verbalization is a key process that can help develop self-regulated learning among children» (p. 362), [« les études démontrent que la verbalisation peut améliorer l'apprentissage par les enfants de l'information, des actions modélisées et des stratégies, ainsi que leur efficacité personnelle pour effectuer des tâches. Collectivement, ces résultats soutiennent que la verbalisation est un processus clé qui peut aider à développer un apprentissage autorégulé chez les enfants] ".

La verbalisation oriente l'attention des élèves sur les caractéristiques pertinentes de la tâche et peut aider ceux-ci à maintenir une attitude positive vis-à-vis de celle-ci. L'utilisation de la verbalisation améliore les apprentissages et la capacité des élèves à coder, à stocker et à mémoriser les données et ainsi faciliter leur récupération et l'utilisation ultérieures.

Les discussions, verbalisations et débats entre élèves ou entre élèves et enseignant se fondent sur une verbalisation explicite et partagée. De ce point de vue, des questions restent à clarifier en revenant sur les fondements sur lesquels le débat d'idées repose ainsi que sur la validité de son utilisation. Par exemple, quand l'insérer dans le processus didactique ? Caverni (1988) a examiné la verbalisation en tant que source observable d'informations sur les processus cognitifs. Compte tenu du moment où elle se produit en ce qui concerne l'exécution de la tâche, il distingue trois types de verbalisation : la verbalisation préalable à l'exécution (en considérant ce qui sera ou doit être fait), les verbalisations concomitantes (en considérant ce qui est fait dans l'action) et les verbalisations consécutives (en considérant ce qui a été fait). Quelle peut être la longueur de telles délibérations ? Concernant la durée du débat d'idées, les recherches (Chang, 2006 ; Zerai, 2011) montrent qu'il ne doit pas excéder plus de deux à trois minutes pour être efficace. Enfin comment, par qui et dans quelles conditions ce débat peut-il être géré ? II faut laisser le dialogue se dérouler, de façon indépendante, le plus possible mais si le professeur désire vraiment faire un apport dans ce débat, son intervention doit être brève et concise (Zerai \& Mekni, 2017 ; Zghibi, 2013) en évitant de parachuter des solutions relevant d'un prêt-à-porter didactique. 
Mais pourquoi avoir recours à la verbalisation? II apparaît que l'étude de celle-ci devrait fournir aux élèves des informations sur les obstacles rencontrés dans leurs efforts pour résoudre les tâches imposées par le jeu. Ces informations peuvent être utilisées par l'enseignant ou être partagées entre les élèves tout en débattant des moyens appropriés pour effectuer les tâches à venir.

\section{Quelques recherches à propos du débat d'idées}

II est à noter que la plupart des recherches que nous allons présenter sont de type descriptif et ont pour objectif de décrire des faits, des événements, des comportements, en utilisant différentes stratégies d'observation. Au-delà de leurs objectifs de description, ces recherches ont le plus souvent une visée compréhensive et d'explication (Astolfi, 1993). Une des premières études sur l'enseignement comparé dans les sports collectifs a été effectuée à la cité scolaire d'Avallon (Gréhaigne, 1994 ; Gréhaigne, Richard, \& Griffin, 2005). Ce travail envisageait de tenter de répondre à la question suivante : y-a-t-il une différence significative entre les deux types d'enseignement (approche technique, approche tactique) concernant le développement des capacités à jouer chez des élèves de collège ? Les résultats ont montré que les élèves ayant eu une approche tactique en relation avec le jeu et des débats (qui ne s'appelaient pas encore débats d'idées) ont plus progressé que les élèves qui ont été confrontés à une approche technique du jeu. À la lumière de cette expérience, l'approche tactique semble produire de meilleurs résultats en termes de performances de jeu si un temps suffisant lui est consacré. Cette approche offre aux élèves une éducation plus sûre et plus durable concernant la prise de décision face à différents problèmes tactiques (Turner \& Martinek, 1995).

Pour sa thèse, Chang (2009) a conçu un plan de recherche pour étudier les effets du débat d'idées sur les élèves taïwanais de cours moyen 2. Deux classes de 32 élèves chacune ont été sélectionnées pour l'étude sur la base de niveaux similaires d'expérience de jeu en basket-ball (72\% et $73 \%$ débutants respectivement). Pour les deux groupes, la session de basket-ball a duré 6 semaines, avec trois leçons de 40 minutes par semaine, pour un total de 18 leçons. Alors qu'une approche technique a été utilisée avec le groupe témoin, le groupe expérimental a été exposé à une approche constructiviste qui comprenait des débats d'idées de 5 minutes après des matchs de 5 minutes dans des jeux réduits.

Concernant les statistiques de jeu, contrairement au groupe témoin, le groupe expérimental a affiché un nombre de balles jouées et un nombre de tirs au panier significativement plus importants dans le post-test, par rapport au pré-test. En ce qui concerne la lecture du jeu dans les situations standardisées, alors que les groupes étaient comparables au prétest, le 
groupe expérimental a obtenu des résultats significativement supérieurs dans le post-test dans 6 situations sur dix. II n'y a pas de différence significative dans le groupe témoin. Enfin, l'analyse qualitative du contenu du débat d'idées a montré une augmentation progressive d'une compréhension mutuelle entre les élèves et l'utilisation des règles d'action tout au long de l'expérience.

Zghibi (2009) a effectué une étude dans laquelle, le protocole de recherche utilisé a consisté en l'organisation d'un cycle de football de huit leçons avec 80 élèves du premier cycle du secondaire (15-16 ans), répartis dans quatre classes différentes. Les situations proposées à 5 x 5 sont des situations de jeu, sur un terrain de handball. Chaque séance était composée de deux situations de jeu (deux matchs), sous le contrôle de l'enseignant, entrecoupées par une séquence de verbalisation assurée toujours par le même enseignant, qui pose des questions ouvertes de types: Que s'est-il passé ? Comment expliquer ce qui s'est passé ? Comment faire pour progresser ? Chaque classe (douze garçons et huit filles) est divisée en deux groupes ( 1 et 2 ) de dix élèves. Les groupes 1 et 2 sont constitués de cinq joueurs et de cinq observateurs Après le jeu, les élèves chargés de l'observation sont impliqués dans le débat d'idées.

Les variables d'observation sélectionnées ont été les balles jouées (BJ), les balles conquises $(B C)$, les balles perdues $(B P)$, les tirs $(T)$ et les buts marqués $(B)$. Après ce premier match, un débat d'idées de 6 minutes est organisé entre les équipes $A$ et $B$ tout comme pour les équipes $\mathrm{C}$ et D. Au terme du cycle d'apprentissage, une progression « négligeable » a été observée avec des critères non significatifs.

Des résultats plus intéressants sont ressortis de l'analyse qualitative du contenu du débat. II y a peu de réponses hors sujet au cours des huit leçons car la décision avec «justification » est restée stable et il y a une augmentation notable de la catégorie justification tout au long du cycle. En ce qui concerne la présence ou l'absence des enseignants aux débats, les résultats ont montré qu'une plus forte argumentation des élèves a été observée lorsque l'enseignant n'était pas présent lors du débat. Les chercheurs ont conclu qu'en l'absence de leur enseignant, les élèves se sentaient plus à l'aise pour exprimer leurs pensées même si cela signifiait pousser leurs idées à l'extrême.

Zerai (2006), dans son expérimentation, note, pour les joueuses n'ayant pas eu de débat d'idées (groupe A), une progression au niveau technique et une tentative d'application directe des consignes sans même les interpréter ou les adapter aux caractéristiques du rapport de forces. Dans le groupe $B$, l'observation des comportements des joueuses après un débat d'idées, marque un changement au niveau de l'organisation du jeu, comme par exemple une montée de balle rapide et organisée ou la recherche d'une récupération rapide 
de la balle après la perte de celle-ci. La différence au niveau des apprentissages entre les deux groupes met en lumière l'efficacité de cette approche dans la construction du jeu. La méthodologie de débat d'idées constitue l'occasion de mettre en place, à partir d'un problème posé par le jeu, une réflexion collective qui amène, souvent, une amélioration des connaissances tactiques mais également des compétences motrices.

Lafont, Proeres et Vallet (2007) ont mené une recherche auprès de 30 élèves du primaire (cours moyen 1 et 2 ; garçons et filles avec un groupe expérimental et un groupe témoin) à l'aide d'un jeu réduit en basket-ball. Trois équipes équilibrées de 5 élèves pour chaque condition ont été fixées sur la leçon 1; des pré-tests ont été administrés lors de la leçon 2. Dans chacune des cinq leçons suivantes, les 15 sujets expérimentaux ont joué 3 matchs de 7 minutes par leçon, en alternance avec des débats d'idées de 6 minutes (une équipe débattait pendant que les deux autres jouaient leur match). L'enseignant a réglé le débat, stimulant la discussion en cas de besoin et s'assurant que chaque participant parlait à son tour. Les résultats ont montré des différences significatives en faveur du groupe expérimental et, selon ces auteurs, ont démontré « la valeur des échanges verbaux en petits groupes coopératifs, non pas pour les compétences individuelles avec le ballon mais dans le contexte d'un jeu collectif plus adapté » (p. 469).

Dans une étude plus récente (Harvey \& Gittins, 2014), des enregistrements vidéo ont également été utilisés pour fournir des commentaires aux élèves au moment de leurs débats d'idées. Trois groupes d'élèves de 13 à 14 ans du secondaire ont été constitués $(n=34$ : $12,12,10$ ) et ont reçu un enseignement de football pendant 6 leçons. Le groupe 1 (6 vs 6 ) a reçu un retour vidéo (V-BF) pour les trois premières leçons, puis on l'a supprimé. Le groupe 2 (6 vs 6) a eu des V-BF ajoutées sur les leçons de 4 à 6, tandis que le groupe 3 a été utilisé comme groupe témoin. Dans chaque leçon, les équipes ont participé à des entraînements centrés sur le jeu pendant 20 minutes en 5 vs 5 . Ensuite, les équipes ont engagé un débat d'idées de 20 minutes, discutant des aspects de leur performance sur la base de l'enregistrement vidéo de leur séance d'entraînement précédente. La discussion s'est concentrée sur la prise de décision tactique.

Les deux autres équipes expérimentales se sont engagées dans un débat d'idées sans enregistrement vidéo de leur séance d'entraînement préalable. Trois variables du Team Sport Assessment Procedure [(TSAP) (Gréhaigne et al., 1997; Gréhaigne et al., 2005)] ont été utilisées comme variables dépendantes : balle jouée, indice d'efficacité et score du match. Une augmentation marquée et significative des performances apparaît à la semaine 3 , puis chute de manière significative lorsque les joueurs n'ont plus de V-BF. Les résultats pour le groupe 2 ont montré un effet miroir du V-BF: leurs balles jouées sont restées basses 
jusqu'à la semaine 3, puis ont bondi de manière spectaculaire et significative montrant, parlà, d'incontestables progrès.

Enfin, Lenzen et Reylé (2017) posent le problème de la relation complexe entre la verbalisation au travers de la formulation de règles d'action et leur concrétisation dans le jeu. La méthodologie repose sur l'analyse a priori des situations d'apprentissage. Les résultats obtenus auprès de deux jeunes enseignants d'éducation physique sont contrastés. La recherche porte précisément sur l'évolution des comportements prometteurs des élèves avec les connaissances/règles d'action émergeant lors des débats d'idées et autres moments institutionnalisés par l'enseignant. L'analyse de la chronologie de ces événements potentiels comme la dévolution d'un problème à résoudre aux élèves, la formulation de règles d'action par les élèves et/ou l'enseignant, etc. constituent de ce fait un enjeu important de cette recherche. Les résultats sont conformes aux données de travaux antérieurs montrant que la formulation de règles d'action lors de débats d'idées ne se concrétise pas nécessairement immédiatement dans l'action motrice des élèves.

Finalement dans ces recherches, malgré des cycles d'apprentissage assez courts et des preuves partielles, on peut noter un effet positif du débat d'idées. L'examen de la littérature s'est limité à l'utilisation du questionnement et du débat dans de petits groupes d'élèves en sport collectif mais comme nous l'avons déjà vu, plus avant, le débat concerne d'autres activités physiques voire d'autres disciplines, en particulier en mathématique ou en sciences.

\section{Débat scientifique ou débat d'idées}

Dans un débat scientifique, débattre, c'est, d'abord, se parler, argumenter, donner son opinion, proposer des réponses, mais c'est aussi apprendre à écouter, apprendre à exprimer des idées, apprendre à défendre ses arguments... C'est ce travail de délimitation du champ des possibles qui fait émerger des contraintes et des besoins et constitue, en fait, une appropriation du problème à résoudre. Cela permet aux élèves (Charlot, Lecorre, Legrand, Leroux, \& Di Martino, 2015 pour une revue) de construire peu à peu un raisonnement, qui, dès lors, n'apparaît plus comme parachuté mais, au contraire, comme partie intégrante des réponses à apporter. Une telle construction fait apparaître toute l'importance du travail argumentatif et du débat en classe puisque c'est de la discussion des arguments au sein de l'équipe et de l'expérimentation sur le terrain qu'émergeront des connaissances à examiner et stabiliser.

Le débat en sport collectif est donc une discussion organisée, une confrontation d'opinions à propos du jeu. Il suit une séquence de jeu qui a été observée et dont le contenu est analysé 
et délibéré par les membres de l'équipe car en éducation physique, il ne peut pas y avoir, entre élèves, de débat scientifique, stricto sensu, en raison du manque de connaissances scientifiques suffisamment valides. C'est pourquoi, nous avons choisi de parler de « débats d'idées » même si ce terme idée peut apparaître comme un peu abstrait mais, comme sa définition le souligne, est bien représenté dans l'esprit, par opposition aux phénomènes concernant l'affectivité ou l'action (CNRTL, 2020).

Ici, la compétence à communiquer ne renvoie pas uniquement à prendre la parole mais aussi à entendre une interrogation, faire des propositions crédibles, éviter de blesser verbalement et enfin à soutenir une controverse. En ce domaine, l'apport heuristique des propositions faites par les élèves est intéressant car il concerne les organisations opérationnelles, tactiques et/ou stratégiques ou il renouvelle les problématiques du traitement des relations d'opposition in vivo. Après une pratique régulière du débat, on observe un changement d'attitudes. II n'est pas rare de voir des élèves exiger de définir un objet jusque là mal précisé ou la preuve d'une évidence énoncée par un autre élève (Charlot et al., 2015).

Dans un débat en EPS, la réflexion ne doit pas concerner seulement l'acquisition des compétences motrices car les situations offrent un contexte concret pour explorer une stratégie, prévoir une nouvelle feuille de route ou un ensemble de règles d'action à utiliser. Au cours d'un cycle d'apprentissage, les élèves doivent pouvoir mettre en évidence leurs succès et leurs échecs ainsi qu'échanger avec leurs pairs et l'enseignant sur des actions efficaces ou non. Un exemple de débat nous est fourni par Zerai (2017) à la fin d'une rencontre de handball perdue par l'équipe $A$.

JIHEN: - On a perdu. (Donnée $\left.{ }^{\circ}{ }^{\circ}\right)$

AWEL: - On n'a pas réussi à marquer (Complétant la donnée $n{ }^{\circ 2}$ )

Le professeur : Que s'est-il passé dans le jeu ?

JIHEN: - L'équipe $B$ a fait une bonne défense de zone. (Donnée $n{ }^{\circ} 3$ )

MARWA: - Nous aussi, mais trop de dribbles. (Donnée $n^{\circ} 4$ prenant appui sur donnée $n$ ०3)

KHAWLA: - Par conséquent, chacun de nous voulait être le meilleur. (Donnée $n^{\circ}$ 5)

JIHEN: - Ce n'est pas seulement ça, on bouge tous dans la même direction pour récupérer le ballon et ensuite on ne peut pas avancer parce que nous sommes au même endroit. (Donnée $n^{\circ} 6$ reprenant les données $n^{\circ} 4$ et $n^{\circ} 5$ )

MARWA: - L'équipe $B$ est mieux organisée que nous. (Donnée $n{ }^{\circ} 7$ ) 
Les élèves doivent être en mesure de prendre en compte plusieurs aspects d'une question par une analyse critique de leurs pensées et des points de vue en présence. Ils/elles doivent être capables d'envisager diverses perspectives fondées sur des aspects objectifs en relation avec la capacité à apprécier d'autres points de vue. Cet ensemble est important de manière à pouvoir travailler et partager avec des personnes ayant des perceptions différentes.

Dans l'enseignement des sports d'équipe, l'utilisation de la verbalisation et le développement du débat d'idées dans les classes répond à divers besoins. Cela permet, dans l'équipe, de constituer un cadre de référence commun, de construire, sur le plan conceptuel, des règles d'action (Gréhaigne, 1989), des règles de gestion de l'organisation du jeu ainsi que de développer la pensée critique à propos des conditions de jeu. Pour que cela fonctionne bien, il faut envisager, rapidement, un bilan des connaissances, des conceptions et des représentations qui préexistent chez les élèves (Zerai, 2006 ; Zerai \& Mekni, 2017). En effet, dans une recherche (Zerai, 2011), des jeunes filles tunisiennes de classe de première qui n'avaient jamais joué aux sports collectifs et qui débutaient un cycle de handball restèrent muettes lors des premiers débats d'idées proposés. Il faudra plusieurs leçons pour que, l'activité et l'expérience aidant, des discussions puissent se tenir. Cependant, concernant les débutant(e)s en sport collectif, le débat d'idées est un lieu privilégié pour assimiler les règles et leur utilisation. Pour obtenir un débat utile, il a fallu laisser aux élèves le temps de construire un minimum de connaissances et de vocabulaire pour mettre en mot les aspects tactiques et techniques du jeu. Une fois ce langage spécifique un peu maitrisé, la verbalisation a pour fonction explicite de favoriser la prise de conscience et la conceptualisation de ce qui a été fait, aidant les filles et les garçons à analyser leur propre pratique et à communiquer sur elle. A la fin du cycle de travail, Zerai (2018) a montré, en utilisant un questionnaire, que les filles du lycée de Thala avaient apprécié le débat d'idées en classe. Elles ont estimé que les débats avaient contribué à améliorer leur pensée tactique et leur capacité de communication orale. En outre, comme l'ont exposé les élèves, les débats ont permis de mieux appréhender les connaissances et compétences motrices à apprendre, de renforcer leur confiance en elles et d'améliorer leurs aptitudes au travail d'équipe. Ainsi, contrairement aux résultats classiquement issus des recherches en sociologie, les effets du débat d'idées ne paraissent pas corrélés de façon significative à l'origine sociale (Poggi \& Moniotte, 2011 ; Poggi, Musard, \& Wallian, 2007). Cependant, l'adhésion à une forme de pratique nouvelle avec des débutantes ou des débutants nécessite du temps pour que les élèves affinent leurs activités et les pratiques culturelles qui leur donnent du sens (Dietsch, Brière-Guénoun, \& Wane, 2015). La place de 
l'élève dans le système enseignement / apprentissage, sa façon de raisonner et la manière d'envisager ses représentations sont ici essentielles. Les résultats d'autres études (Park, Kier \& Jugdev, 2011) suggèrent que, comparés aux élèves qui n'utilisent pas le débat et l'apprentissage en coopération, les élèves qui apprennent en coopération progressent plus vite et que cet apprentissage interactif améliore la mémorisation, ainsi que la capacité de raisonnement critique. Cette posture réflexive est une posture mentale qui n'est pas spontanée et le débat d'idées qui l'utilise relève bien de la volonté de promouvoir les méthodes actives en pédagogie.

\section{Verbalisation, pensée, pratique réflexive et pensée tactique}

Prise dans un sens large, la pensée est une activité mentale, en grande partie consciente, qui recouvre l'ensemble des fonctions psychiques et psycho-physiologiques ayant la connaissance pour objet (CNRTL, 2019). Ces processus permettent à l'être humain d'être au contact de la réalité matérielle et sociale tout en élaborant des concepts et les reliant entre eux pour apprendre, créer et agir. Dans un groupe, c'est aussi une représentation collective, un ensemble d'idées, une façon de juger ainsi qu'une façon de vivre et/ou de penser.

La conscientisation possède, certes, ses propres limites mais une bonne partie de la cognition peut être explorée. Métacognition (Flavell, 1979 ; Godbout, 2003) et pratique réflexive sont des processus mentaux qui visent à apprendre à partir d'expériences vécues, par un retour de la conscience sur son propre fonctionnement, dans le but de prendre conscience de ses manières d'agir et de réagir, dans les situations de la vie. La pratique réflexive sur le jeu consiste à caractériser la manière dont l'action est organisée, à décoder ce qui sous-tend l'action et à formaliser ses connaissances pour les rendre lisibles et compréhensibles par tous (Mc Bride, 1991; Gréhaigne \& Godbout, 1998). Plus précisément, le débat aide les apprenants à développer une pensée réflexive et critique en examinant des arguments, en collectant des informations, en effectuant des analyses, en remettant en question des projets, en évaluant les résultats de l'action. Le développement d'une pensée et d'une pratique réflexive s'articule notamment autour d'une pensée critique et créative, de compétences argumentatives et d'habiletés métacognitives (Godbout, 2003). Les élèves recourent à la pensée critique, dans un contexte donné, pour mieux distinguer, parmi les informations qu'ils reçoivent, celles qui sont les plus pertinentes au regard des buts qu'ils ou elles poursuivent. Distanciation, abstraction et interlocutions deviennent des pièces maîtresses d'une approche socio-sémio-constructiviste (Wallian \& Gréhaigne, 2004 ; Gréhaigne, Wallian, \& Brière-Guenoun, 2015) de la didactique des sports collectifs. 


\section{1. Pensée critique, pratique réflexive}

Si les enseignants veulent utiliser le débat et la pensée critique dans leurs classes, il est important de développer une définition pouvant être appliquée aux supports pédagogiques (et expliquée aux élèves) de manière pratique. Malgré la grande diversité de définitions, il existe un large consensus sur les compétences cognitives nécessaires pour devenir un «penseur critique » performant. Ainsi Facione (1990) propose une taxonomie avec six grandes catégories: interprétation, analyse, évaluation, inférence, justification et autorégulation. Chaque catégorie est subdivisée en étapes spécifiques impliquée dans la recherche, la préparation et la réalisation d'un débat (Tableau I). Cette liste des compétences cognitives fournit un cadre pratique pour analyser les progrès et réguler la pensée critique dans les débats. II est à noter que cette taxonomie n'est pas sans rappeler la taxonomie du domaine cognitif proposée par Bloom et ses collaborateurs en 1956.

Tableau I. Les compétences cognitives d'un «penseur critique » performant (Facione, 1990).

\begin{tabular}{|l|l|}
\hline Compétences & Étapes spécifiques \\
\hline 1. Interprétation & Catégorisation, décodage de la signification, clarification du sens \\
\hline 2. Analyse & $\begin{array}{l}\text { Examiner des idées, identifier des arguments, analyser des } \\
\text { arguments }\end{array}$ \\
\hline 3. Évaluation & Évaluer les demandes, évaluer les arguments \\
\hline 4. Inférence & $\begin{array}{l}\text { Interroger les preuves, envisager des alternatives, tirer des } \\
\text { conclusions }\end{array}$ \\
\hline 5. Justification & $\begin{array}{l}\text { Constater les résultats, justifier les procédures, présenter des } \\
\text { arguments }\end{array}$ \\
\hline 6. Autorégulation & Auto-examen, autocorrection \\
\hline
\end{tabular}

La pensée réflexive (Pallascio \& Lafortune, 2000) repose essentiellement sur la notion de prise de conscience de ses propres connaissances, hypothèses et expériences passées. Ainsi les expériences passées constituent des informations nécessaires dans les prises de décisions. II faut cependant prendre en compte le fait qu'un ou une élève accumule de l'expérience tout au long de sa vie et, de ce fait, sa capacité de réflexion va évoluer. La pensée réflexive est le fait de faire l'expérience de quelque chose, de penser à cette expérience, pour finalement apprendre grâce à cette expérience et agir en conséquence face à un problème. La pensée réflexive est, par ailleurs, une partie du processus de la pensée critique. En effet, la pensée critique fait référence à la capacité d'analyser les informations de manière la plus objective possible afin de porter un jugement. Cela implique 
l'évaluation des données, des faits, des phénomènes observables et les résultats de la recherche. Ainsi, la pensée critique exige que l'on utilise sa capacité à raisonner. II s'agit donc d'être un apprenant actif plutôt qu'un destinataire passif d'informations. En bref, cela signifie que dans le processus de la pensée critique, il faut remettre en question les idées reçues, les sentiments, les opinions personnelles et même les informations jusqu'à ce qu'il y ait des preuves concrètes qui justifient leur véracité. Ainsi, la pensée réflexive et la pensée critique sont intrinsèquement liées. II est cependant utile de préciser que la pensée critique est un terme générique qui englobe un grand nombre de pensées. En revanche, la pensée réflexive, qui fait directement référence à la réflexion, est un processus plus clair et plus précis qui permet de donner un sens à une expérience, une pratique. La pensée réflexive implique notamment des éléments analytiques et critiques qui peuvent être articulés à travers des formes d'expressions parlées, écrites ou autres, aboutissant ainsi à ce que l'on appelle la pratique réflexive. Cela signifie que la pensée réflexive n'est pas uniquement destinée à des considérations abstraites, mais essentiellement à des utilisations dans des situations réelles et concrètes. Dans le jeu et dans les débats, la conceptualisation est bien au service de l'action en cours, en vue d'une théorisation à court terme qui offre au joueur une "structure conceptuelle de la situation »(Vidal-Gomel \& Rogalski, 2007). Celle-ci utiliserait des relations causales entre concepts (invariants opératoires, règles d'action, règles de l'organisation du jeu) en relation avec les indicateurs de prise d'information ou de décision. Cette structure conceptuelle permet aux acteurs de construire une représentation mentale de la situation, orientée vers l'action qui se déroule et se transforme dans le temps et avec le temps.

\section{2. Pratique réflexive et débat d'idées}

Le débat d'idées dans le "modèle d'apprentissage des décisions tactiques 》 (Gréhaigne, Billard, \& Laroche, 1999) renvoie à une pensée critique coopérative qui vise à concilier sa propre position et celle de l'autre partie; elle cherche à s'appuyer sur les idées des uns et des autres pour que chacun puisse bénéficier de l'engagement (Tan, 2017). Dans le contexte du débat d'idées, cela implique des compétences cognitives telles qu'interpréter, observer, inférer, analyser, évaluer, formuler des propositions, créer et prendre des décisions en fonction du contexte; rechercher des informations pertinentes et fiables; être adaptable et flexible face aux changements.

On peut supposer que la pratique réflexive et la pensée critique sont à l'œuvre pendant le débat d'idées et que c'est, d'abord, une réflexion coopérative qui est organisée. Chez les élèves, le débat les encourage à mieux s'approprier le contenu de la leçon, car ils y 
participent activement, de manière globale, profonde et personnelle. II les forme également à évaluer et discuter les données qu'ils reçoivent quotidiennement. Le recours à la pratique réflexive implique, également, une visée émancipatrice qui devrait aider les élèves à se libérer des manipulations de tous ordres. En ce sens, "le savoir s'oppose à l'ignorance, la foi ou la croyance et est formulable en une proposition dont on admet la vérité pour des raisons intellectuelles et communicables » (Monteil, p 24).

À partir des protocoles verbaux, l'activité mentale identifiable chez les élèves ou les joueurs, permettant d'inférer leur pensée tactique, peut renvoyer soit à des intentions référées à un but ou à un état futur. A ce stade, passer d'avoir des intentions à prendre des initiatives en jeu, c'est passer de la stratégie à la tactique, c'est quitter le domaine de la réflexion pour aller vers une application concrète de la pensée tactique.

\section{3. Pensée tactique}

La pensée tactique se construit et se nourrit à partir d'outils cognitifs. Bien sûr, la tactique doit être considérée aussi dans une unité dialectique avec la technique. Mais c'est la pensée qui donne un contenu à la tactique des procédés techniques en les transformant en actions. Pendant le match, la pensée, dénommée conventionnellement pensée tactique, possède un caractère actif, favorisant l'initiative. Cette forme d'intelligence engagée dans la pratique doit combiner à la fois le flair, l'attention vigilante, le sens de l'opportunité. Dans le jeu, l'accent est toujours mis sur " l'efficacité pratique », en un mot la recherche du succès et de la réussite dans le domaine concret de l'action. Ainsi, toute activité de jeu est un acte forcément tactique, quel que soit le niveau où se situe le joueur, car il consiste à résoudre, pratiquement et dans le respect des règles du jeu, un grand nombre de problèmes posés par l'opposition. Dans cette complexité, Mouchet, Amans-Passaga et Gréhaigne (2010) proposent de distinguer différents niveaux d'abstraction qui consistent à :

- réfléchir collectivement sur les données concrètes de la situation en liaison étroite avec la perception et le jeu ;

- utiliser la pensée tactique liée au jeu mais dépassant la situation concrète. Ceci opère un rapprochement entre la situation telle qu'elle a été reconnue et des règles, des principes, des réponses ;

- recourir à une pensée tactique abstraite qui n'est pas directement liée à l'acte de jeu mais qui s'opère à l'aide de représentations figurées ou de généralisations abstraites pour apporter des réponses aux problèmes posés.

Ces apprentissages s'effectuent soit par imprégnation soit par des exercices appropriés, il faut alors se poser la question de l'apprentissage de la pensée tactique car une véritable 
formation à la pensée tactique évite aux élèves d'apprendre et de jouer schématiquement. La capacité à observer, à caractériser et à interpréter ce qui se passe dans une rencontre devient alors primordiale pour tous.

Dans les sports collectifs, le développement d'une pensée tactique autonome (Gréhaigne \& Caty, 2014 ; Menaut, 1998), permettant d'analyser et d'organiser les réponses face à des problèmes analogues, consiste en l'acquisition et la stabilisation de connaissances, de compétences motrices et d'expériences tactiques. Souvent, chez ces élèves, le développement d'une pensée tactique passe par la possibilité de s'acculturer des pratiques dont ils ignorent le sens et ne maîtrisent ni les techniques, ni les tactiques (Poggi, 2016). Ce développement de la pensée tactique passe aussi par la mise en mots que les élèves vont utiliser lors du débat d'idées. Dans cette mise en mots, le langage ne fait pas que décrire le jeu qui lui serait préexistant, c'est lui qui délimite ce que les joueuses et les joueurs peuvent en penser et en dire (Nachon \& Chang, 2004). Enfin, la possibilité pour un joueur de s'investir dans le jeu repose sur la reconnaissance du rôle déterminant, parce que première chez l'être humain, de la pensée abstraite organisatrice de l'activité. Pour nous, cela recouvre la construction d'une pensée tactique qui assure aux joueurs une représentation juste et maniable en plein jeu des rapports et des effets d'opposition. Ici, il n'y a pas d'incompatibilité entre initiatives individuelles et actions collectives, mais au contraire aucune action collective n'est possible sans une initiative individuelle de tous les joueurs et à tout moment. La base de l'efficacité en sport collectif consiste à permettre, à tous les participants d'une même équipe, le plein déploiement de l'initiative individuelle avec comme support d'une pensée tactique partagée. La construction de cette véritable intelligence tactique semble si importante qu'elle nécessite une conception renouvelée de la formation des joueurs.

Dans cette perspective, l'approche sémio-socio-constructiviste des apprentissages en sport collectif (Wallian \& Gréhaigne, 2004) met l'accent sur l'activité du sujet, ses observations et leur verbalisation ainsi que sa capacité à bâtir des projets pour apprendre.

\section{Conclusion}

Dans ce type de pédagogie centrée sur l'élève, on vise le déclenchement d'une activité adaptative orientée vers la recherche de solutions motrices plus efficaces. Mais, un véritable débat à visée d'apprentissage en EPS consiste bien à organiser une activité de classe qui ne convoque pas uniquement des connaissances déclaratives. Ainsi, les interlocutions dans le débat visent à analyser la pratique et à proposer des réponses adaptées dans un cadre précis et situé : l'affrontement en cours. Dans cette forme de collaboration et de 
communication, il faut, d'une part, expliciter le rôle de chacun et, d'autre part, inscrire ces tâches dans une temporalité en vue de leur exécution. Le langage aide à structurer la pensée et à la construction-transmission de connaissances en invitant les joueurs ou les joueuses à analyser le résultat des situations d'apprentissage proposées.

Les joueurs, les observateurs et l'enseignant doivent être capables de tenir leur rôle sans trop troubler l'ordre scolaire habituel. L'hypothèse est que jouer et observer permettront aux élèves de progresser dans le jeu. L'utilisation des jeux à effectif réduit offrira aux apprenants davantage d'occasions de s'exercer et d'acquérir les compétences in situ. La réflexion des élèves sur leur jeu, leurs succès et leurs erreurs peut nourrir leur pensée tactique. En un sens, ce qui est en jeu, c'est l'apprentissage et le développement des connaissances tactiques en action, en relation forte avec l'apprentissage des compétences motrices. Néanmoins, il faut noter que la mise au point d'actions en projet, sous forme d'une feuille de route, provoque parfois la proposition de circulations fantaisistes du ballon et des joueurs. En outre, il faut considérer que si le jeu n'est pas un enjeu significatif pour les élèves et si ceux-ci ne s'inscrivent pas dans un projet commun, les apprentissages sont vraiment faibles, voire absents... A l'opposé, si l'enjeu proposé représente un pas en avant trop important pour certains élèves, ceux-ci décrochent et ne veulent plus participer...

Concernant le métier d'enseignant, une des difficultés majeures rencontrées par le professeur lorsqu'il donne la parole aux élèves est de rester suffisamment neutre pour laisser ceux-ci s'exprimer. Enfin, l'enseignant peut rencontrer également des difficultés d'ordre méthodologique car ce type d'approche des sports collectifs nécessite des connaissances et des attitudes inhabituelles qui doivent être précises et mesurées pour ne pas court-circuiter le processus et les débats. Cependant, l'occasion donnée aux joueurs de pratiquer plusieurs tâches dans des cycles longs évite la monotonie et donne aux élèves le temps nécessaire pour stabiliser de nouvelles ressources.

\section{Bibliographie}

Astolfi, J.-P. (1993). Trois paradigmes pour les recherches en didactique. Revue Française de Pédagogie, 103, 5-18

Bloom, B.S., Engelhart, M.D., Furst, E.J., Hill, W.H., \& Krathwohl, D.R. (1956). Taxonomy of Educational Objectives. Handbook I: The Cognitive Domain. New York: David McKay.

Bonnery, A., \& Cadopi, M. (1990). Danse. In AEEPS (Ed.), Éducation physique et didactique des APS (pp. 51-62). Paris : France: AEEPS. 
Caty, D. (2004). Des compétences gymniques aux compétences artistiques : vers une modélisation de l'acrosport scolaire. Thèse de doctorat (non publiée) en Sciences du langage, Didactique, Sémiotique. Besançon : Université de Franche-Comté.

Caverni, J.-P. (1988) La verbalisation comme source d'observables du fonctionnement cognitif. In J.-P. Caverni, C. Bastien, P. Mendelsohn, \& G. Tiberghien, G. (Eds.), Psychologie cognitive : modèles et méthodes (pp. 253-273). Grenoble : Presses Universitaires.

CNRTL. Centre national de ressources textuelles et lexicales (2019). Pensée. Consulté sur : http://www.cnrtl.fr/definition/pensée.

CNRTL. Centre national de ressources textuelles et lexicales (2020). idée. Consulté sur : http://www.cnrtl.fr/definition/idée.

Charlot, G., Lecorre, T, Legrand, M., Leroux, A., \& Di Martino, H. (2015) Le débat scientifique en classe : une démarche d'investigation collective pour une culture scientifique commune. In L. Theis (Ed.), Pluralités culturelles et universalité des mathématiques: enjeux et perspectives pour leur enseignement et leur apprentissage (pp. 847-860). Actes du colloque EMF 2015 - GT10.

Darnis, F., \& Lafont, L. (2008). Effets de la dissymétrie de compétence pour un apprentissage coopératif en dyades en éducation physique et sportive. Les Cahiers Internationaux de Psychologie Sociale, 79, 69-83.

Darnis, F., \& Lafont, L. (2015). Cooperative learning and dyadic interactions: Two modes of knowledge construction in socio-constructivist settings for team-sport teaching. Physical Education and Sport Pedagogy, 20, 459-473.

Darnis, F., Lafont, L., \& Menaut, A. (2007). Interactions verbales en situation de coconstruction de règles d'action au hand-ball: L'exemple de deux dyades à fonctionnement contrasté. eJRIEPS, 11, 56-17.

Deriaz, D., Poussin, B., \& Gréhaigne, J.-F. (1998). Le débat d'idées. Revue EP\&S, 273, 8082.

Dietsch, G., Brière-Guénoun, F., \& Wane, T. (2015). Forme de pratique scolaire du football en EPS, débats d'idées et apprentissages en milieu difficile. Recherches en éducation, 23, 117-131.

Flavell, J. H. (1979). Metacognition and cognitive monitoring - A new area of cognitivedevelopmental inquiry. American Psychologist, 34, 906-911.

Godbout, P. (2003). Reflection on innovation and renovation: Authentic assessment and subject matter expertise in Physical Education. In L. Sena Lino, R. Trindade Ornelas, F. Carreiro Da Costa, \& M. Piéron (Eds.), Innovations and new 
technologies in physical education, sport, research and/on teacher and coach preparation (Cagigal Lecture). Proceedings CD-ROM of the AIESEP Congress, Universidade da Madeira, 22-25 November 2001.

Gréhaigne, J.-F. (1989). Football de mouvement. Vers une approche systémique du jeu. Thèse (non publiée). Dijon : Université de Bourgogne.

Gréhaigne, J.F. (1994) Analyse comparative de deux types d'enseignement des sports collectifs : approche centrée sur la technique et approche centrée sur le jeu. Rapport de Recherche. IUFM de Franche-Comté.

Gréhaigne, J.-F., Billard, M., \& Laroche, J.Y. (1999). L'enseignement des jeux sportifs collectifs à l'école. Conception, construction, évaluation. Bruxelles : De Boeck. (Collection Sciences et Pratiques du Sport).

Gréhaigne, J.-F., \& Caty, D. (2014). Développer la pensée tactique à l'école. In J.-F. Gréhaigne (Ed.), L'intelligence tactique. Des perceptions aux décisions tactiques en sports collectifs (pp. 279-300). Besançon : Presses de l'Université de FrancheComté.

Gréhaigne, J.-F., \& Deriaz, D. (2007). Le débat d'idées. In J. F. Gréhaigne (Ed.), Configurations du jeu, débat d'idées \& apprentissage du football et des sports collectifs (p. 111-119). Besançon : Presses universitaires de Franche-Comté.

Gréhaigne, J.-F., \& Godbout, P. (1998). Observation, critical thinking and transformation: three key elements for a constructivist perspective of the learning process in team sports. In R. Feingold, R. Rees, G. Barrette, L. Fiorentino, S. Virgilio, \& E. Kowalski (Eds.), Education for life (pp. 109-118). New-York: Adelphi University.

Gréhaigne, J.F., J.-F. Richard, \& Griffin, L. (2005). Teaching and learning team sports and games. New York: Routledge Falmer.

Gréhaigne, J.-F., Wallian, N., \& Brière-Guenoun, F. (2015). Débat d'idées, langage, interactions discursives et apprentissage des sports collectifs. In J.-F. Gréhaigne, \& G. Dietsch (2015). Quelques aspects théoriques de la didactique des sports collectifs. Préparation aux concours de recrutement (pp. 54-78). eJRIEPS, HorsSérie $N^{\circ} 1$.

Harvey, S., \& Gittins, C. (2014). Effects of integrating video-based feedback into a teaching games for understanding soccer unit. Agora for Physical and Education and Sport, 16, 271-290.

Lab, F. (2007). Comment «réhabiliter » le demi-fond pour motiver les élèves à courir? Thèse de doctorat (non publiée) en Science du sport. Besançon : Université de Franche-Comté. 
Lafont, L., Proeres M., \& Vallet, C. (2007). Cooperative group learning in a team game: role of verbal exchanges between peers. Social Psychology of Education, 10, 93-113

Lenzen, B., \& Reylé, J. (2017). Des savoirs planifiés par les enseignants aux connaissances construites par les élèves en éducation physique. Nouveaux Cahiers de la Recherche en Éducation, 20(1), 36-60.

McBride, R. E. (1991). Critical thinking - An overview with implications for physical education. Journal of Teaching in Physical Education, 11, 112-125

Menaut, A. (1998). Le réel et le possible dans la pensée tactique. Talence: Presses Universitaires de Bordeaux.

Mouchet, A., Amans-Passaga, C., \& Gréhaigne, J.-F. (2010). L'approche technologique. In M. Musard, M. Loquet \& G. Carlier (Eds.), Sciences de l'intervention en EPS et en sport. Résultats de recherches et fondements théoriques (pp. 201-222). Paris: Éditions EP\&S.

Nachon, M. \& Chang, C.W. (2004). Interactions verbales entre les élèves et projet d'action en Basket-ball en classe de troisième. eJRIEPS, 5, 15-36.

Pallascio, R. \& Lafortune, L. (Eds.) (2000). Pour une pensée réflexive en éducation. Québec : Presses de l'Université du Québec.

Park, C., C. Kier, \& Jugdev, K. (2011). Debate as a teaching strategy in online education: A case study. Canadian Journal of Learning and Technology, 37(3), 1-17.

Poggi, M.-P. (2016). Handball scolaire pour les filles en EPS. Quels risques d'inégalités ? In J.-F. Gréhaigne (Ed.), Les objets de la technique. De la compétence motrice à la tactique individuelle (pp. 271-286). Besançon : PUFC.

Poggi, M.-P., \& Moniotte J. (2011). Quelle place pour le sociologique dans les recherches en didactique de l'EPS ? Éducation et didactique, 5(1), 29-44.

Poggi, M.-P., Musard, M., \& Wallian, N. (2007). Approche sociologique des interlocutions en EPS : le cas du basket-ball. Communication orale présentée au colloque international du Pôle Nord-Est des IUFM Les effets des pratiques enseignantes sur les apprentissages des élèves. Besançon.

Schunk, D. H. (1986). Verbalization and children's self-regulated learning. Contemporary Educational Psychology, 11, 347-369.

Tan, C. (2017). Teaching critical thinking: Cultural challenges and strategies in Singapore. British Educational Research Journal, 43, 988-1002.

Turner, A. P., \& Martinek, T.J. (1995). Teaching for understanding: A model for improving decision making during game play. Quest ,47, 44-63. 
Vidal-Gomel, C. \& Rogalski, J. (2007). La conceptualisation et la place des concepts pragmatiques dans l'activité professionnelle et le développement des compétences. @activité(s), 4(1), 49-84.

Wallian, N., \& Gréhaigne, J.-F. (2004). Vers une approche sémio-constructiviste des apprentissages moteurs. In G. Carlier (Ed.), Si l'on parlait du plaisir d'enseigner l'éducation physique (pp. 167-179). Montpellier : AFRAPS.

Zerai, Z. (2006). Comment les filles apprennent-elles en Handball ? Apport de la verbalisation. Mastère en didactique des Activités Physiques et Sportives. Tunis : ISEFC.

Zerai, Z. (2011). Apprentissage du handball chez les jeunes filles tunisiennes et françaises ; apport de la verbalisation. Thèse de doctorat (non publiée) en Sciences du Sport. Université de Franche-Comté, Besançon.

Zerai, Z. (2017). Language interactions and learning in team sport in Tunisia. Journal of Physical Education Research, 4(I), 27-38.

Zerai, Z. (2018). Verbalization and Learning in Handball. Advances in Physical Education, 8, 7-19

Zerai, Z., \& Mekni, R. (2017). How to evaluate interactions during a debate of ideas. Creative Education, 8(4), 539-548.

Zghibi, M. (2009). Interactions langagières des élèves et apprentissage en football : le cas de quatre classes de 9ème Année de Base en Tunisie. Thèse de Doctorat (non publiée) en Sciences du Sport, Université de Franche-Comté. 